\title{
Edme Antoine Durand (1768-1835) : un bâtisseur de collections
}

Edme Antoine Durand (1768-1835): a builder of collections

\section{Louise Detrez}

\section{OpenEdition}

Journals

Édition électronique

URL : http://journals.openedition.org/cel/485

DOl : $10.4000 /$ cel. 485

ISSN : 2262-208X

Éditeur

École du Louvre

Édition imprimée

Date de publication : 1 avril 2014

Référence électronique

Louise Detrez, «Edme Antoine Durand (1768-1835) : un bâtisseur de collections », Les Cahiers de I'École du Louvre [En ligne], 4 | 2014, mis en ligne le 01 avril 2014, consulté le 30 avril 2019. URL : http:// journals.openedition.org/cel/485; DOI : 10.4000/cel.485

\section{(c) (i) $\odot$}

Les Cahiers de l'École du Louvre sont mis à disposition selon les termes de la licence Creative Commons Attribution - Pas d'Utilisation Commerciale - Pas de Modification 4.0 International. 


\title{
Cahiers de l'École du Louvre
} recherches en histoire de l'art, histoire des civilisations archéologie, anthropologie et muséologie

Numéro 4. Avril 2014

\author{
Edme Antoine Durand (1768-1835) : \\ un bâtisseur de collections \\ Louise Detrez
}

Article disponible en ligne à l'adresse :

http://www.ecoledulouvre/cahiers-de-l'ecole-du-louvre/numero4avril2014/Detrez.pdf

Pour citer cet article :

Louise Detrez, «Edme Antoine Durand (1768-1835) : un bâtisseur de collections », Cahiers de l'École du Louvre, recherches en histoire de l'art, histoire des civilisations, archéologie, anthropologie et muséologie [en ligne] n 4, avril 2014, p. 45 à 55.

\section{Ccreative commons \\ $\circledast \circledast$}

(C) École du Louvre

Cet article est mis à disposition selon les termes de la Licence Creative Commons Attribution - Pas d'utilisation commerciale - Pas de modification 3.0 non transposé. 


\section{Cahiers de l'École du Louvre recherches en histoire de l'art, histoire des civilisations archéologie, anthropologie et muséologie \\ Numéro 4. Avril 2014}

Sommaire

Éditorial

p. 1

\section{Étude}

Le musée de Sculpture comparée au prisme de la collection de cartes postales éditées par les frères Neurdein (1904-1915)

Dominique Jarrassé et Emmanuelle Polack

p. $2-20$

Dossier:

Les modalités de la collecte : rapt, troc, marché, fouilles, don...

Et leur impact sur l'objet

Dans collection, il y a collecte...

Introduction de Dominique Jarrassé.

p. $21-23$

Entrer en collection. Pour une ethnographie des gestes et des techniques de collecte

Julien Bondaz

p. $24-32$

De la meilleure façon de constituer une collection. Le cas des émaux «byzantins » de Mikhaill Botkine

Aglaé Achechova.

Edme Antoine Durand (1768-1835) : un bâtisseur de collections

Louise Detrez p. $45-55$

Réunir une documentation pour l'Art Brut : les prospections de Dubuffet dans l'immédiat après-guerre au regard du modèle ethnographique Baptiste Brun p. $56-66$

Travailler sur des objets en faisant se confronter les points de vue : regards croisés sur le langage fou : l'exemple des écrits bruts

Vincent Capt p. $67-75$

D’un regard-pilote à l'écart. L'impact de Dubuffet sur les collectes de l'association L'Aracine

Déborah Couette. p. $76-86$

La cabane éclatée. Morcellement des objets immobiliers apparentés à l'art brut

Roberta Trapani .p. $87-95$ 


\section{Edme Antoine Durand (1768-1835) : un bâtisseur de collections ${ }^{1}$}

\section{Louise Detrez}

Fils d'un riche négociant en vins d'Auxerre bientôt ruiné par la politique monétaire révolutionnaire, E. A. Durand (fig. 1) s'engage dans un négoce lucratif qui le place dès l'âge de trente ans à la tête d'une fortune considérable. Marchand de tableaux patenté de 1817 à 1822 , puis qualifié de négociant ou d'antiquaire, le chevalier Durand assemble des collections dont les contemporains ne parlent qu'avec force hyperboles. "Célèbre collecteur d'antiquités ${ }^{2}$ " en son temps, illustre inconnu aujourd'hui, son nom reste toutefois attaché à deux cabinets d'antiquités d'exception : la "première » collection Durand est vendue en bloc en 1825 pour former le noyau du musée Charles X, la « seconde » est dispersée lors d'une vente aux enchères après sa mort, en 1836.
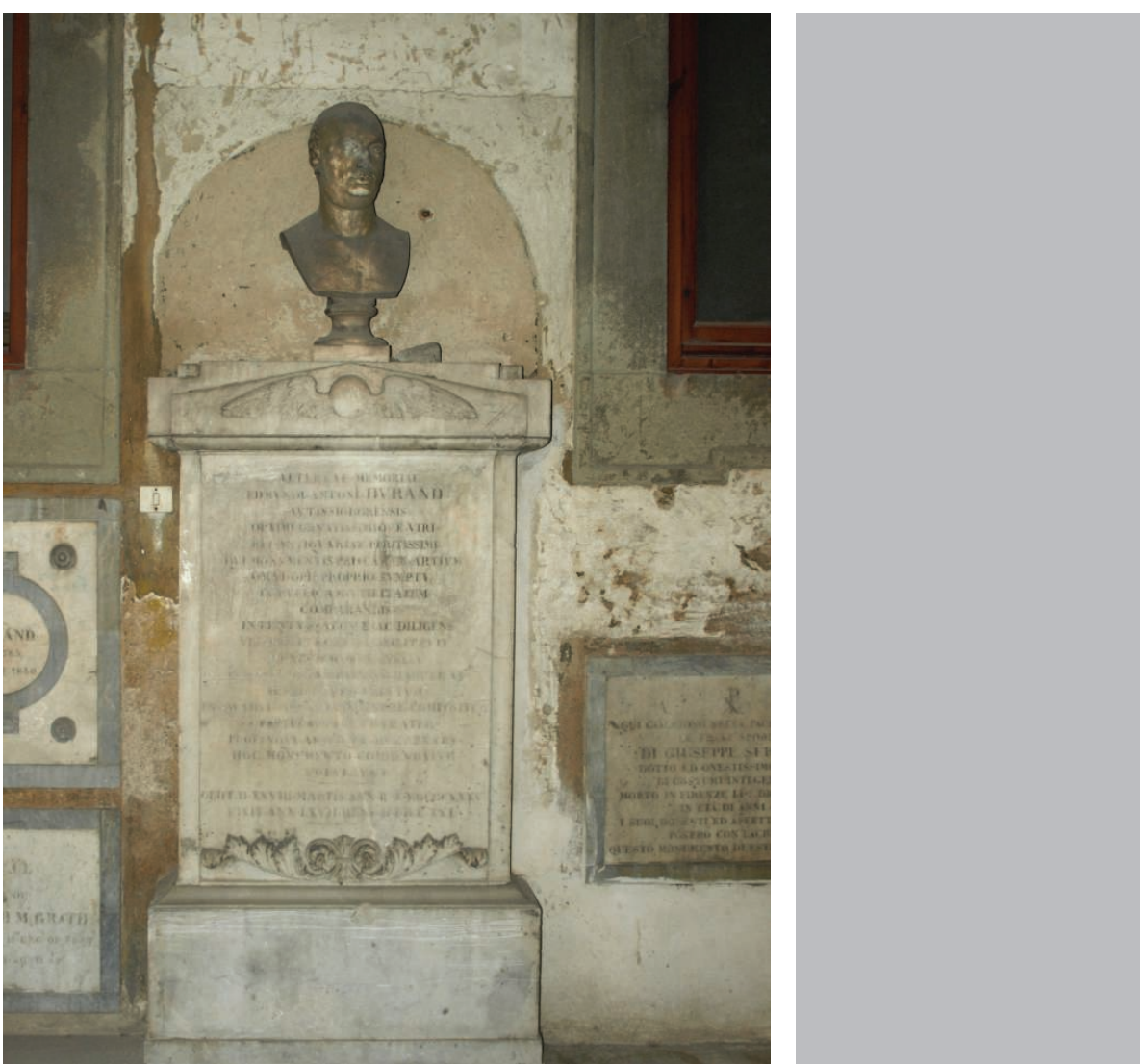

L'examen du cas du chevalier Durand pourrait ne pas déparer un recueil consacré aux modalités de la collecte. Recherchant le meilleur dans des domaines courus, ce collectionneur sillonne l'Europe sans regarder à la dépense ${ }^{3}$ ni contrevenir aux lois

1. Nos plus vifs remerciements vont à Brigitte Bourgeois, Dominique Jarrassé, Violaine Jeammet et Anne Ritz-Guilbert.

2. Journal des débats, 12 avril 1835 , p. 2.

3. Son propre témoignage (lettre d'E. A. Durand au comte de Forbin, le 16 novembre 1824 Archives des Musées Nationaux 76, 1832) comme une anecdote savoureuse (le chevalier Durand traverse la Manche pour tancer I'antiquaire italien Campanari qu'il accuse de brader ses vases antiques à Londres, lui achète sa marchandise bien au-dessus du prix demandé et s'en retourne sur le continent en sa compagnie : Ian Jenkins, « La vente des vases Durand (Paris 1836) et leur réception en Grande-Bretagne », actes du colloque Anticomanie. La collection d'antiquités auX XVIII et XIXe siècles, Montpellier-Lattes, 9-12 juin 1988, publiés sous la direction de Krzystof Pomian et Annie-France Laurens, éditions de l'EHESS, Paris, 1992, pp. 271-272 et Bodil Bundgaard Rasmussen, « London... in reality the capital of Europe "P.O. Brøndsted's dealings with the British Museum" », actes du colloque Peter Oluf Brøndsted (1780-1842). A Danish Classicist in his European Context, Copenhague, 5-6 octobre 2006, publiés sous la direction de Bodil Bundgaard Rasmussen, Jorgen Steen Jensen, John Lund et Michael Märcher, Copenhague, 2008, pp. 152154) le prouvent. 
en vigueur ${ }^{4}$. Marchand lui-même, il s'approvisionne essentiellement sur le marché et destine une partie au moins de ses trouvailles à la revente ${ }^{5}$. Amateur éclairé estimé de la communauté archéologique européenne ${ }^{6}$, ses achats d'importance sont relayés dans la presse érudite et souvent assortis d'éloges 7 . Sa pratique de la " collecte ", celle tout à la fois d'un collectionneur averti et d'un marchand avisé, atteste un flair hors pair et une extraordinaire aptitude à l'appropriation.

Cette étude voudrait rendre compte de l'étendue de la passion du chevalier Durand pour la collection. Après avoir brossé un bref portrait du collectionneur qu'était E. A. Durand, nous discuterons la pertinence des appellations de "première » et « seconde » collection Durand qu'a consacrées l'usage : contredites par les faits, elles masquent en réalité un pan considérable de sa curiosité.

\section{Un « antiquaire d'élite $»^{8}$}

Un choc esthétique reçu en 1799 en Italie où le chevalier Durand était employé à la suite de l'armée révolutionnaire semble être à l'origine de son dévouement à l'art'. Car un investissement total, aux deux sens du terme, le caractérise : son épitaphe révèle qu'il "passa sa vie, rendit son nom célèbre en mettant autant de soin que de zèle à acheter des monuments des arts du passé à ses propres frais, $\mathrm{y}$ consacrant toute sa fortune, pour l'intérêt public ${ }^{10}$ » et un acte notarié posthume résume sa biographie à ce seul trait : " $\mathrm{M}^{\mathrm{r}}$ Durand n’a jamais été marié. Tout dévoué à la science archéologique, il avait placé sa fortune presque entière dans les objets d'art et d'antiquité qui composaient son cabinet ${ }^{11}$. "

Une exigence d'excellence aura en effet constamment sous-tendu ses démarches : le marchand Nicolas Bénard rapporte ainsi que vers 1820, ayant porté " au point de perfection " sa collection d'estampes, " $\mathrm{M}^{\mathrm{r}} \mathrm{E}$. D. ${ }^{* *}$ se voyait arrêté dans sa plus grande jouissance, celle de s'enrichir chaque jour de quelques pièces remarquables ", mais qu'il fut bientôt pris du « désir d'élever au plus haut degré possible ses Collections d'antiquités " ${ }^{12}$. Aussi le dit-on lancé dans une quête perpétuelle de l'objet rare ${ }^{13}$, sillonnant l'Europe et courant les ventes publiques ${ }^{14}$.

\section{Les deux collections Durand}

\section{Le noyau du musée Charles $X$}

Le cabinet Durand jouit dans les années 1820 d'une réputation internationale : "Depuis longtemps, la collection de $\mathrm{M}^{\mathrm{r}}$ Durand, l'une des plus considérables et des mieux choisies qu'il y ait en Europe, a mérité l'admiration des connoisseurs par son étendue et sa variété ${ }^{15}$ ", écrit le comte de Clarac. Véritable "panorama des $\operatorname{Arts}^{16}$ ", ses quelque 7400 objets ont abondé les actuels départements des

4. En témoignent ses demandes d'exportation adressées aux autorités napolitaines, voir infra.

5. Il cède par exemple une partie des antiques de Joséphine au comte de Pourtalès-Gorgier, cat. d'exp., De Pompéi à Malmaison, les antiques de Joséphine, sous la direction de Sophie DescampsLequime et Martine Denoyelle, Rueil-Malmaison, 22 octobre 2008-27 janvier 2009, Musée du Louvre éditions, Paris, 2008, note 72 p. 25.

6. Les Français J.-F. Champollion, C. Lenormant, T. E. Mionnet et D. Raoul-Rochette, le Belge J. de Witte, les Allemands E. Gerhard et T. Panofka ou encore le Danois P. O. Brøndsted fréquentent son cabinet.

7. Par exemple Bullettino dell'Istituto di corrispondenza archeologica, 1830, pp. 94, 257, 262$263 ; 1831$, p. $185 ; 1833$, pp. 224,$255 ; 1834$, p. 9.

8. Catalogue d'une belle collection de tableaux capitaux et de curiosité formant le cabinet de feu M. Durand-Duclos, Paris, 1847, avertissement non paginé.

9. S[imon] Ph[ilippe] Ch[audé], Notice sur feu M. le chevalier Durand, Paris, Didot, 1836, pp. 6-7.

10. Épitaphe latine composée par Désiré Raoul-Rochette, traduite avec l'aide de Jean-Paul Brachet; idem p. 6.

11. Liquidation de la succession, AN, ET/XXVI/1075.

12. Nicolas Bénard, Catalogue de la précieuse collection d'estampes recueillie par Mr E. D.**, Paris, 1821, p. IV.

13. Bullettino dell'Istituto di corrispondenza archeologica, $n^{\circ} 7,1835$, p. 48.

14. En témoigne notamment l'inventaire de sa bibliothèque, AN, ET/XXVI/1066.

15. Lettre du comte de Clarac au comte de Forbin, 18 novembre 1824, AMN, Z6, 1832.

16. Lettre du comte de Forbin au vicomte de La Rochefoucauld, 19 novembre 1824 dans Louis Courajod, «La collection Durand et ses séries du Moyen Âge et de la Renaissance au musée du Louvre », Bulletin monumental, 1888, p. 341. 
Antiquités grecques, étrusques et romaines, des Antiquités égyptiennes et des Objets d'art du musée du Louvre.

Le fonds grec, étrusque et romain n'est pas le premier cabinet d'antiques du chevalier Durand : dès 1806, Aubin-Louis Millin qualifiait sa collection de vases antiques de "peu nombreuse, mais bien choisie ${ }^{17}$ "; en 1812, A. Clener écrivait au même Millin que Joseph Tôchon venait d'acquérir ses plus beaux vases ${ }^{18}$; enfin, son premier achat documenté, la célèbre amphore de Chantilly acquise auprès de Nicola Vivenzio en 1801, avait été vendue au comte de Pourtalès-Gorgier en $1813^{19}$. E. A. Durand, ensuite, s'approvisionne aux meilleures sources, selon les modes d'acquisition les plus divers. Depuis Athènes, Louis Sébastien Fauvel converti au commerce d'antiquités lui procure des vases ${ }^{20}$. De ses périples outremonts, E. A. Durand rapporte des antiques par centaines ${ }^{21}$ : marchands de haut vol, tel Raffaele Gargiulo ${ }^{22}$ ou James Millingen ${ }^{23}$, et collectionneurs, comme Gabriele Judica ${ }^{24}$, sont ses fournisseurs. À Paris, les ventes publiques sont l'occasion d'acquérir bronzes ${ }^{25}$ et vases antiques ${ }^{26}$. Surtout, à partir de 1814 , le chevalier Durand s'adjuge les antiques de Joséphine que dispersent ses héritiers, et notamment les pièces du musée de Portici exhumées à Pompéi et Herculanum offertes en 1802 par le roi de Naples Ferdinand IV ${ }^{27}$. Des achats sont également conclus avec des amateurs parisiens : avant 1824, E. A. Durand acquiert une partie de l'imposante collection de vases que le comte de Paroy avait constituée à Naples dès $1787^{28}$, tandis que Salomon Reinach envisage une transaction avec J. Tôchon ${ }^{29}$. Sa réception est enthousiaste : on loue alors la " rareté absolue ${ }^{30}$ " des antiques de Portici, célèbre l'une des plus belles collections de vases en Europe par la variété de son répertoire formel et son extension chronologique ${ }^{31}$ et considère que " la plus belle suite de monumens antiques en bronze, qui ait existé en France ${ }^{32}$ " se signale par l'intérêt iconographique de sa plastique et la valeur documentaire de ses instrumenta.

«Les objets égyptiens sont tous de premier choix, et à peu près les plus beaux parmi tout ce qui a été envoyé à Paris ${ }^{33}$ ». Nul besoin, en effet, d'inférer un voyage en Égypte : en 1822 par exemple, E. A. Durand acquiert aux ventes Saulnier et Thédenat-Duvent bijoux, papyri, amulettes, "faïences », métaux, statuettes en

17. Aubin-Louis Millin, Monuments antiques ou nouvellement expliqués, Paris, vol. II, p. 102. 18. Lettres d'A. Clener à A.-L. Millin, 12 août et 5 octobre 1812, BnF, Mss Fr 24681, fo291 et fo 292. Nous remercions Florence Le Bars-Tosi de nous avoir signalé ces documents.

19. Martine Denoyelle et Dietrich von Bothmer, «Naturalisme et illusion : les Vases grecs et étrusques, une œuvre d'Alexandre-Isidore Leroy de Barde (1777-1828) », Revue du Louvre, vol. 2, 2002, p. 39 ; cat. d'exp., De l'Égypte à Pompéi, le cabinet du duc d'Aumale, Ludovic Laugier, Chantilly, musée Condé, 5 juin-9 septembre 2002, Paris, Somogy, 2002, p. 42.

20. Alessia Zambon, « Fauvel et les vases grecs », Journal des savants, 2006, vol. 1, pp. 54-56. 21. En 1824, par exemple, il achète trois cents pièces pour $45000 \mathrm{~F}$., lettres d'E. A. Durand au comte de Forbin, novembre et 16 novembre 1824, op. cit. note 16, pp. 336-339.

22. Andrea Milanese, « Raffaele Gargiulo (1785-après 1870) restaurateur et marchand d'antiquités. Notices sur le commerce des vases grecs à Naples dans la première moitié du XIX siècle », actes du colloque El vaso griego en el arte europeo de los siglos XVIII y XIX, Madrid, 14 et 15 février 2005, publiés sous la direction de Paloma Cabrera et Pierre Rouillard, Madrid, 2007, pp. 71-72.

23. Florence Le Bars-Tosi, « James Millingen (1774-1845), le "Nestor de I'archéologie moderne" », dans Manuel Royo, Martine Denoyelle et al., Du voyage savant aux territoires de l'archéologie. Voyageurs, amateurs et savants à l'origine de l'archéologie moderne, Paris, de Boccard, 2011, pp. $179-180$.

24. Lettre d'Adrien de Jussieu à Stendhal, 11 janvier 1838, Stendhal, Correspondance (18351842), Paris, Gallimard, 1968, vol. III, pp. 545-546. Au sujet de G. Judica voir Michael Kiene, Die Alben von Jakob Ignaz Hittorff. Die italienische Reise 1822-1824 (Paris-Rom), Cologne, Schriften der Universitäts und Stadtbibliothek Köln, 2012, pp. 97-98.

25. Ventes Van Hoorn Van Vlooswyck 1809, Campion de Tersan 1819, Grivaud de la Vincelle 1820 et Castex 1822.

26. Ventes Saulnier 1822, Montfort et autres provenances 1823, Isabelle Decise, Les ventes aux enchères à Paris dans la première moitié du XIXe siècle. Le cas des antiquités grecques, étrusques et romaines, mémoire d'étude de l'École du Louvre présenté sous la direction de Corinne Jouys Barbelin et Néguine Mathieux, 2012, vol. 3, pp. 11, 59.

27. Cat. d'exp. cité note 5 , pp. 21-25 et 30-38.

28. Laureen Bardou, Jean-Philippe Guy Le Gentil, comte de Paroy (1759-1824) : portrait d'un collectionneur de vases grecs entre l'Ancien Régime et l'Empire, mémoire de recherche de I'École du Louvre présenté sous la direction de Brigitte Bourgeois et Martine Denoyelle, 2011, pp. 45-62 et 110-169.

29. Salomon Reinach, Peintures de vases antiques recueillies par Millin (1808) et Millingen

(1813), Paris, Firmin Didot et compagnie, 1891, p. 96, note 1.

30. S. P. Chaudé, op. cit. note 9, p. 11 .

31. Lettre citée note 3.

32. Claude-Madeleine Grivaud de la Vincelle, Recueil de monumens antiques, la plupart inédits, découverts dans I'ancienne Gaule, Paris, 1817, vol. I, pp. XIV-XV.

33. Bulletin des sciences, 1825, pp. 131-132. 
bois, vases en albâtre, momies, sarcophages et statues de pierre. La collection Durand, qui compte de précieux jalons de l'art des Moyen et Nouvel Empire ainsi que de l'époque ptolémaïque ${ }^{34}$, a alors si bonne presse qu'elle rend inutile aux yeux du comte de Clarac l'achat de la seconde collection Salt ${ }^{35}$.

Majoliques, terres vernissées, émaux et vitraux, suscitent enfin l'admiration : "on ne conçoit pas que l'on ait pu en rassembler un aussi grand nombre des formes les plus belles et des dessins les plus riches et les plus variés, on ne sait ce qu'on doit admirer le plus ou de leur magnificence ou de leur conservation ${ }^{36}$ ", s' exclame le comte de Clarac. Les majoliques conservent la mémoire des compositions des grands maîtres italiens, le fonds français rend un hommage mérité à l'art national ${ }^{37}$, tandis que l'ensemble revêt une valeur inaugurale dans les collections nationales ${ }^{38}$, se félicite-t-on.

Trois arguments semblent justifier aux yeux de l'administration l'acquisition de la collection Durand, le 2 mars 1825. D'abord, panser les blessures du passé que constituent les restitutions consécutives au traité de Vienne ${ }^{39}$ et le refus de la collection Drovetti ${ }^{40}$. Ensuite, prévenir l'exportation d'une collection Durand convoitée $^{41}$. Enfin, ouvrir le règne de Charles X par un bienfait pour la nation ${ }^{42}$ en créant au Louvre un musée sans égal ${ }^{43}$. La transaction est globalement défavorable au vendeur : E. A. Durand demande 500000 F. pour sa collection ${ }^{44}$ que le comte de Pourtalès estime valoir un million ${ }^{45}$, et en obtient 480000 F. en six versements annuels sans intérêts, après y avoir ajouté des centaines d'objets à titre gracieux. Elle n'en demeure pas moins la principale affaire de sa carrière ${ }^{46}$.

\section{Une ultime collection « inappréciable »47}

Au cours de " huit années de recherches et de voyages ${ }^{48}$ ", E. A. Durand bâtit " un nouveau cabinet beaucoup plus curieux que le précédent ${ }^{49}$ ".

D'abord, le chevalier Durand n'avait en réalité pas cédé l'intégralité de son cabinet au Louvre : il conserve "la fleur des suites qu'il avait précédemment possédées $^{50}$ ». Ensuite, l'Italie demeure sa principale source d'approvisionnement. Cinq voyages outremonts sont attestés, en $1825^{51}, 1826-1827^{52}, 1830^{53}, 1833^{54}$ et 1834-1835. Au fait de l'actualité archéologique - il compte parmi les associés correspondants de l'Istituto di corrispondenza archeologica depuis $1832^{55}-$, E. A. Durand se procure auprès des fouilleurs (le prince de Canino, les frères Campanari) et de marchands tel Francesco Depoletti ${ }^{56}$, quantité de vases grecs et de bijoux étrusques exhumés à Vulci, nécropole étrusque dont la fouille commence

34. Monique Kanawaty, «Les acquisitions du musée Charles $X »$, Bulletin de la Société française d'égyptologie, 1985, pp. 35-36; « Vers une politique d'acquisitions : Drovetti, Durand, Salt et encore Drovetti », Revue du Louvre, n 4, 1990, p. 268.

35. Lettre du comte de Clarac au comte de Forbin, AMN, AE, 6, 23 février.

36. Lettre citée note 15.

37. Bulletin des sciences, op. cit. note 33, p. 132.

38. L. Courajod, op. cit. note 16, p. 331.

39. Lettre du comte de Forbin au vicomte de la Rochefoucauld, 19 novembre 1924, L. Courajod, op. cit. note 16 , pp. 339-343.

40. Lettre du comte de Forbin au vicomte de la Rochefoucauld, 19 novembre 1924, L. Courajod, op. cit. note 16 , pp. 339-343; lettre citée note 15.

41. Lettre du comte Turpin de Crissé au comte de Forbin, 8 octobre 1824, L. Courajod, op. cit. note 16 , pp. 333-334

42. Lettre citée note 16 .

43. Lettre citée note 15 .

44. Lettre d’E. A. Durand au comte Turpin de Crissé, 11 octobre 1824, L. Courajod, op. cit. note 16 , pp. 334-336.

45. Lettre d'E. A. Durand au comte de Forbin, 16 novembre 1824, idem, pp. 337-339.

46. Liquidation de la succession, citée note 11 .

47. Jean de Witte, Description des antiquités et objets d'art qui composent le cabinet de feu M. le chevalier E. Durand, Paris, Didot, 1836, p. I.

48. S. P. Chaudé, op. cit. note 9, p. 12.

49. Journal des débats, 12 avril 1835, p 2.

50. S. P. Chaudé, op. cit. note 9, p. 13 ; par exemple, J. de Witte op. cit. note 47, n' 420 et $\mathrm{n}^{\circ} 114$.

51. Lettre d'E. A. Durand au comte de Forbin, $1^{\text {er }}$ juillet 1825, AMN, Z6, 1832.

52. Du même au même, 18 septembre 1826 : ibidem, et Naples, Archivio di Stato, Esteri 6377.

53. Naples, Archivio di Stato, Esteri, 6418.

54. Naples, Archivio di Stato, Esteri, 6454.

55. Lettre d'E. A. Durand aux directeurs de I'Istituto, 10 août 1832, Deutsches archäologisches Institut, Rom, Archiv, II Kasten B26.

56. Marie-Amélie Bernard, «Francesco Depoletti (1779-1854), artiste et restaurateur de vases antiques à Rome vers 1825-1854 », Technè, 2008, nos 27-28, pp. 79-84. 
à livrer les trésors. Ses demandes d'exportation du Royaume de Naples ${ }^{57}$ comme $^{2}$ les mentions fréquentes de provenances dans le catalogue de vente confirment cependant la persistance de son intérêt pour l'Italie méridionale. Les ventes parisiennes, enfin, lui fournissent antiquités égyptiennes, céramiques antiques et médailles ${ }^{58}$.

La dernière collection Durand fait l'objet de louanges unanimes : réconciliant quantité et qualité, elle se classe au premier rang de toutes les collections publiques et privées européennes, musée de Naples exceptét ${ }^{59}$. Ses vases, en particulier, brillent " tant sous le rapport des sujets que sous celui de l'art ": illustrant des thèmes iconographiques d'une variété telle qu'elle commande le plan du catalogue de vente et issus de tous les ateliers producteurs identifiés, ils offrent de "magnifiques peintures ${ }^{60}$.

L'espoir d'E. A. Durand de voir sa dernière collection rejoindre la «première " au Louvre est déçu. Rêvant à la réunion au Louvre des vases antiques des collections publiques parisiennes ${ }^{61}$, il s'assignait l'objectif patriotique de rendre sa collection digne du Musée royal ${ }^{62}$, et destinait de longue date ses vases aux musées royaux ${ }^{63}$, intention réitérée dans son testament ${ }^{64}$. L'administration décline l'offre d'achat formulée par ses héritiers : aussi le cabinet Durand est-il dispersé du 25 avril au 27 mai 1836 au cours d'une vente publique qui fit date.

\section{D'autres collections Durand}

Voyons à présent que les engouements artistiques d'E. A. Durand composent une réalité plus complexe que les deux collections qu'on lui attribue d'ordinaire (fig. 2).

Quatre difficultés sont à signaler : l'effectif des collections Durand recensé n'est pas nécessairement complet; en synchronie, la segmentation des collections n'est pas toujours nette ; en diachronie, les cabinets ne se succèdent pas strictement ; enfin, notre connaissance relative de l'approvisionnement, de la teneur et de la dispersion de chaque cabinet varie. Le recensement qui va suivre conserve donc une part d'arbitraire.

\section{Une mystérieuse pinacothèque}

La première collection Durand semble avoir été composée, à la faveur de voyages, de "tableaux précieux et finis " au sujet desquels notre ignorance est complète ${ }^{65}$. Le prix et la facture des œuvres ainsi que le goût dominant de l'époque pourraient-ils suggérer la piste des écoles du Nord ? Faute d'avoir identifié une vente publique ayant dispersé cet ensemble, faisons l'hypothèse qu'il ait pu rejoindre la boutique de ce marchand de tableaux.

\section{« Un cabinet de dessins précieux »66}

Edme Antoine Durand est bien le vendeur anonyme d'un cabinet de dessins dispersé au début du mois d'avril 1820 : Frits Lugt y voyant un homonyme de notre collectionneur, en citant l'adresse ajoutée à main levée au catalogue qu'il consulte,

57. Naples, Soprintendenza, IX, D1, 3/17 et 3/25, D2, 1/7, 1/20, 5/33 et 25.

58. Ventes Morel d'Arleux (1828), Alessia Zambon, « Fauvel et les vases grecs : un catalogue retrouvé », Journal des savants, 2006, vol. 2, pp. 185-193; Mainvielle-Fodor (1828), I. Decise, op. cit. note 26, pp. 14, 74 ; Révil (1830); F*** (1832), I. Decise, op. cit. note 26, pp. 16, 85-88 ; Hauterive (1832), I. Decise, op. cit. note 26, pp. 16, 85-88 ; Canino (1834) et Gastard (1834).

59. Entre autres, J. de Witte, op. cit. note 47.

60. J. de Witte, op. cit. note 47, pp. I-II.

61. Lettre d'E. A. Durand au comte de Forbin, 9 février 1825, AMN, Z6, 1832.

62. Lettres citées notes 44, 45 ; d’E. A. Durand au comte de Forbin, novembre 1824, AMN, Z6, 1832.

63. Brouillon d'une lettre du comte de Forbin ( ?) au comte de Montalivet, 14 avril 1835, AMN,

Z6, 1832.

64. AN, ET/XXVI/1065.

65. Unique mention, S. P. Chaudé, op. cit. note 9, pp. 7-8.

66. [Alexis Nicolas] Pérignon, Catalogue d'un cabinet de dessins précieux des trois écoles et notamment des meilleurs artistes de l'école moderne, Paris, 1820. 
lève au contraire tout doute à ce sujet ${ }^{67}$. La qualité et la bonne conservation de ces feuilles « des trois écoles et notamment des meilleurs artistes », de Dürer à David, en font la renommée ${ }^{68}$.

\begin{tabular}{|c|c|c|}
\hline Contexte & Contenu & Références \\
\hline $\begin{array}{l}\text { 4-5 avril } 1820 \\
\text { commissaire- } \\
\text { priseur (C-P) } \\
\quad \text { Lacoste }\end{array}$ & $\begin{array}{l}187 \text { lots de dessins } \\
\text { et estampes }\end{array}$ & $\begin{array}{l}\text { - Frits Lugt, Répertoire des } \\
\text { catalogues de ventes publiques } \\
\text { intéressant I'art ou la curiosité } \\
\text { (1826-1860), La Haye, Martinus } \\
\text { Nijhoff, 1953, n' } 9761 \\
\text { - [Alexis Nicolas] Pérignon, } \\
\text { Catalogue d'un cabinet de dessins } \\
\text { précieux des trois écoles et } \\
\text { notamment des meilleurs artistes } \\
\text { de l'école moderne, Paris, } 1820\end{array}$ \\
\hline $\begin{array}{c}\text { 18-24 mars } 1821 \\
\text { C-P Bonnefons de } \\
\text { Lavialle }\end{array}$ & $\begin{array}{c}1023 \text { lots } \\
\text { d'estampes }\end{array}$ & $\begin{array}{l}\text { - Idem, n } 9985 \\
\text { - Procès-verbal (PV) : archives de } \\
\text { Paris, D48/E3/15 } \\
\text { - Nicolas Bénard, Catalogue de la } \\
\text { précieuse collection d'estampes } \\
\text { recueillie par Mr E.D. **, Paris, } \\
1821\end{array}$ \\
\hline $\begin{array}{c}\text { 18-23 janvier } 1830 \\
\text { C-P Bonnefons de } \\
\text { Lavialle }\end{array}$ & 529 lots de militaria & $\begin{array}{l}\text { - Id., } \mathrm{n}^{\circ} 12209 \\
\text { - P-V : archives de Paris, D48/ } \\
\text { E3/24 } \\
\text { - Catalogue des armures et armes } \\
\text { diverses composant la collection } \\
\text { formée originairement par feu M. } \\
\text { le baron Percy et complétée par } \\
\text { M.D.***, Paris, } 1829\end{array}$ \\
\hline $\begin{array}{c}\text { 20-23 janvier } 1834 \\
\text { C-P Bonnefons de } \\
\text { Lavialle }\end{array}$ & $\begin{array}{l}316 \text { lots d'objets } \\
\text { d'art et antiquités }\end{array}$ & P-V : archives de Paris, D48/E3/28 \\
\hline $\begin{array}{l}23 \text { décembre } 1835 \\
\text { C-P Lenormant de } \\
\text { Villeneuve }\end{array}$ & $\begin{array}{l}127 \text { lots d'effets } \\
\text { personnels }\end{array}$ & P-V : archives de Paris, D145/E3/9 \\
\hline $\begin{array}{l}\text { 25-27 janvier } 1836 \\
\text { C-P Lenormant de } \\
\text { Villeneuve }\end{array}$ & $\begin{array}{c}226 \text { lots } \\
\text { d'estampes, } \\
\text { gouaches, } \\
\text { aquarelles, } \\
\text { miniatures, dessins, } \\
\text { livres et bordures }\end{array}$ & $\begin{array}{l}\text { - Id., } \mathrm{n}^{\circ} 14194 \\
\text { - P-V : archives de Paris, D145/ } \\
\text { E3/9 } \\
\text { - Antoine François Piéri-Bénard, } \\
\text { Catalogue d'une collection } \\
\text { d'estampes anciennes, de livres } \\
\text { sur les arts et la littérature, de } \\
\text { gouaches et dessins, provenant du } \\
\text { cabinet de feu M. Edme Durand, } \\
\text { Paris, } 1836\end{array}$ \\
\hline $\begin{array}{c}25 \text { avril-27 mai } \\
1836 \\
\text { C-P Lenormant } \\
\text { de Villeneuve et } \\
\text { Bonnefons de } \\
\text { Lavialle }\end{array}$ & $\begin{array}{c}2704 \text { lots } \\
\text { d'antiquités } \\
\text { égyptiennes, } \\
\text { grecques, étrusques } \\
\text { et romaines, et arts } \\
\text { décoratifs d'époque } \\
\text { moderne }\end{array}$ & $\begin{array}{l}\text { - Id., n० } 14322 \\
\text { - P-V : archives de Paris, D145/ } \\
\text { E3/9 } \\
\text { - Jean de Witte, Description des } \\
\text { antiquités et objets d'art qui } \\
\text { composent le cabinet de feu M. le } \\
\text { chevalier E. Durand, Paris, Didot } \\
1836\end{array}$ \\
\hline
\end{tabular}

67. Frits Lugt, Les marques de collections de dessins et d'estampes, Amsterdam, Vereenigde Drukkerijen, 1921, p. 120

68. A. N. Pérignon, op. cit. note 66, p. III. 


\section{« La collection (...) la plus riche et la plus complète en Estampes rares qui ait été recueillie par un particulier $\gg 69$}

Vingt ans d'acquisitions en vente publique, en boutique et auprès de particuliers dans une Europe désormais pacifiée ont placé E. A. Durand à la tête d'une insigne collection d'estampes ${ }^{70}$. Le propriétaire a mis en œuvre une véritable stratégie d'approvisionnement en publiant dès 1819 le catalogue raisonné de son cabinet, remarquable ouvrage d'érudition autant qu'efficace outil publicitaire : la publication en révèle le mérite et multiplie les opportunités d'acquisition ${ }^{71}$.

E. A. Durand s'était proposé de constituer « l'Histoire générale de l'Art de la Gravure, depuis son origine jusqu'à nos jours ${ }^{72}$ ", dans toutes les écoles, à travers ses chefs-d'œuvre et sur le mode de l'exhaustivité : « il est bien peu d'Estampes remarquables par leur beauté, leur rareté ou leur valeur, qui manquent dans ce cabinet $^{73}$ ". Riche en joyaux " des beaux temps de la renaissance des arts $"^{74}$, ce dernier comptait notamment le nielle " introuvable " d'une Paix de Maso Finiguerra $^{75}$ (fig. 3), plus de deux cents épreuves précieuses de Marc Antonio Raimondi et l'œuvre complet d'Albrecht Dürer « unique, peut-être, pour le choix et la belle conservation des épreuves ${ }^{76}$ ".

Figure 3 Gravure anonyme d'apres Maso Finiguerra, Vierge à l'Enfant entouree d'anges Florence
vers 1450 nielle Vienne, Albertina publiée dans Nicolas Bénard, Catalogue de la précieuse collection d'estampes recueillie par Mrecieuse* collection d'estampes recueillie par

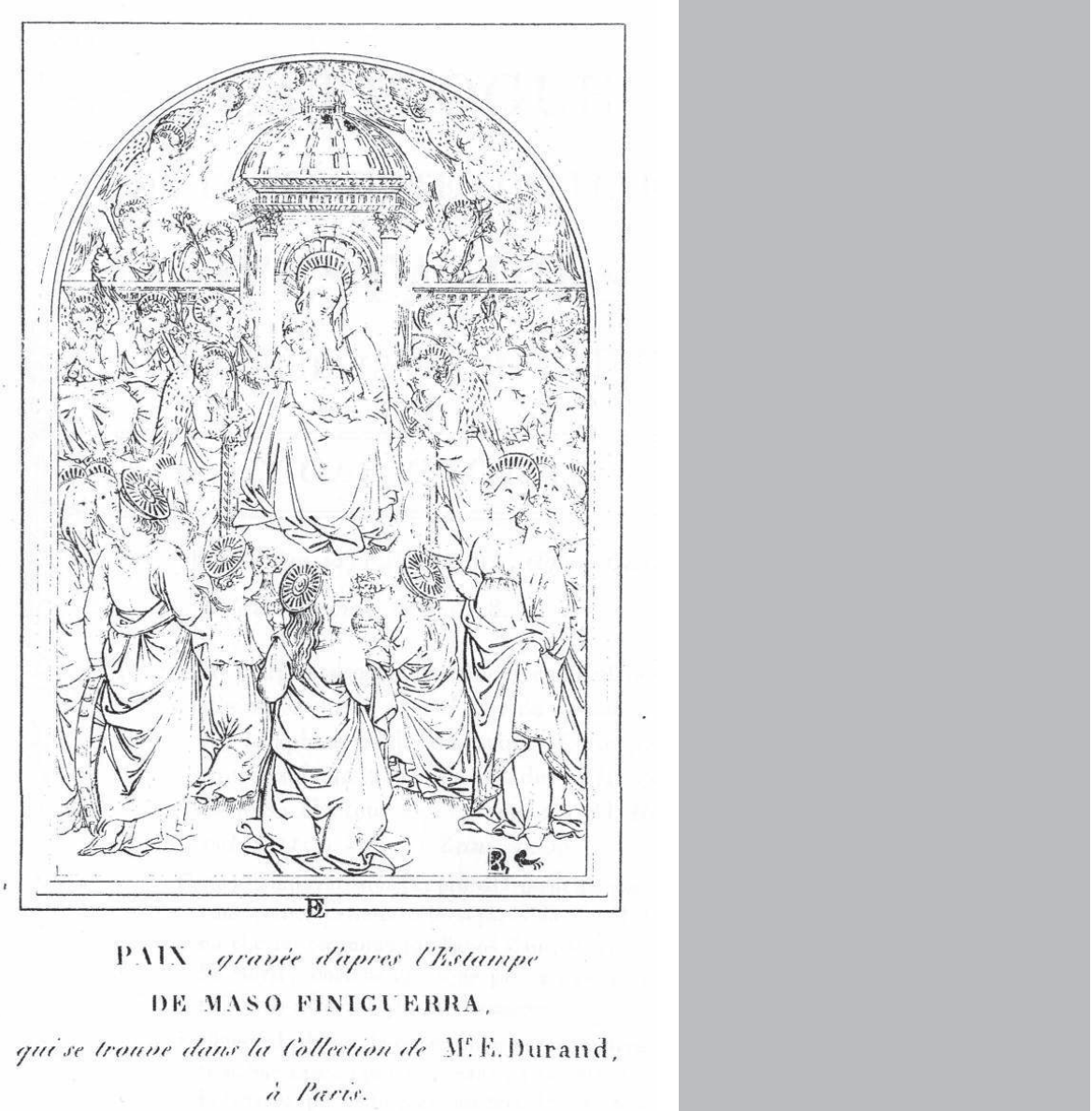

69. N. Bénard, op. cit. note 12, p. III.

70. N. Bénard, op. cit. note 12, p. III et IV; S. P. Chaudé, op. cit. note 9, p. 8.

71. [Edme Antoine Durand], Catalogue des estampes du cabinet E. Durand, Paris, 1819, p. I. 72. Idem, ibidem, p. I et $\mathrm{X}$

73. N. Bénard, op. cit. note 12, p. VII.

74. E. A. Durand, op. cit. note 71 , p. II.

75. Collections Lélu, Borduge, Révil, Durand, (E. A. Durand, op. cit. note 71, p. XIII) et duc de Saxe-Teschen.

76. Joubert père, Manuel de I'amateur d'estampes, Paris, 1821, pp. 274-299 : recensement, prix d'adjudication et commentaire des pièces majeures. 
En 1821, une vente publique destinée à procurer au chevalier Durand les fonds nécessaires à l'enrichissement de sa collection d'antiquités ${ }^{77}$ disperse l'essentiel de cet ensemble.

\section{La passion des gemmes antiques et modernes}

L'étonnant destin des pierres gravées des trésors mêlés de la cathédrale SaintPierre et de la collégiale Saint-Étienne de Troyes offre un exemple éloquent de l'exigence et des méthodes d'E. A. Durand en matière de glyptique antique. Trois cents camées et intailles grecs et romains saisis dans les églises et le palais impérial de Constantinople en 1204 par l'évêque de Troyes et le comte de Champagne, vinrent enrichir les objets liturgiques de ces deux établissements (fig. 4). Réchappées des fontes révolutionnaires, les pierres des deux trésors confondus

Figure 4 :

Gravure des estampages de quelques intailles du trésor de Saint-Etienne de Troyes dans: comte de Caylus, Recueil d'antiquités égyptiennes, étrusques, grecques et romaines V, Paris, 1752, pl. LII. Bibliothèque, collections Jacques Doucet
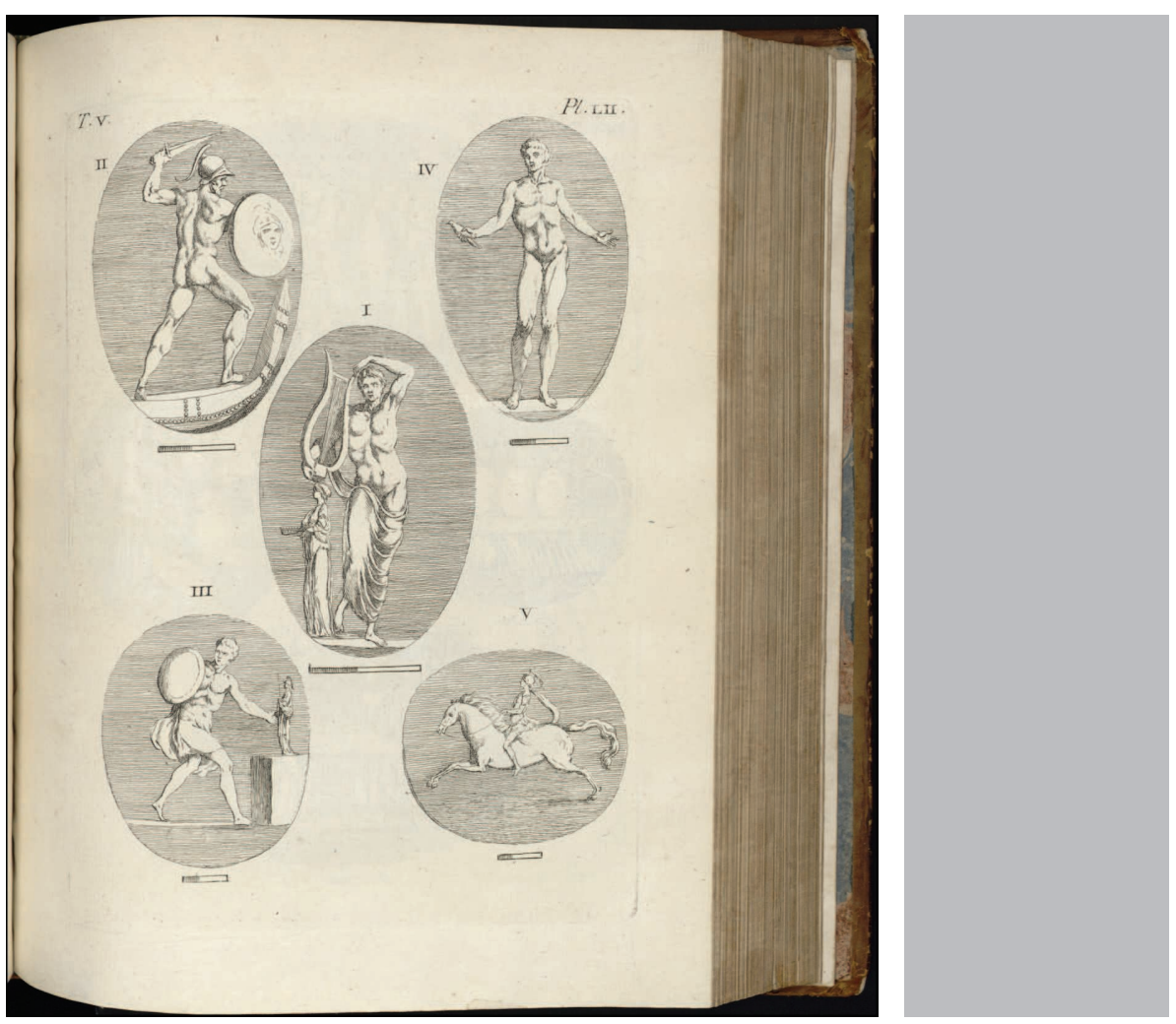

furent restituées en 1807 au chapitre de la cathédrale. Le 8 octobre 1820, la fabrique s'interrogea sur la suite à donner à l'offre d'un amateur parisien adressée à l'abbé de Bonald : acquérir les gemmes pour la somme de $10000 \mathrm{~F}$., nettement supérieure à une précédente estimation. Le lendemain, le paiement était enregistré $^{78}$. Or, l'acquéreur parisien dont Eugène Le Brun-Dalbanne " [est] heureux de ne pas savoir le nom ${ }^{79}$ " n'était autre qu'E. A. Durand. D'abord, l'inventaire de ses papiers mentionne un reçu d'une somme identique, daté du 10 octobre et signé par l'abbé de Bonald, pour le "paiement d'une collection de camées ", précisera l'intéressé lors de la liquidation de la succession Durand ${ }^{80}$. De plus, deux pierres figurent, assorties de leur illustre provenance, au catalogue de la vente après décès du cabinet Durand ${ }^{81}$. On rapporte que leur nouveau propriétaire en avait vendu certaines au détail, avant de céder un lot important à l'Ermitage ${ }^{82}$. Si le client d'E. A. Durand du nom de Köhler, écrivant depuis Saint-Pétersbourg, est bien Heinrich Karl Ernst Köhler (1765-1838), conservateur de l'Ermitage en charge des antiques et garde du cabinet impérial des gemmes, monnaies et

77. N. Bénard, op. cit. note 12, p. IV.

78. Eugène Le Brun-Dalbanne, Les pierres gravées du trésor de la cathédrale de Troyes, Paris, Rapilly, 1880, pp. 9-27.

79. Idem ibidem, p. 26

80. Liquidation de la succession citée note 11

81. J. de Witte, op. cit. note 47, n $^{\text {os }} 2525$ et 2533

82. E. Le Brun-Dalbanne, op. cit. note 78, p. 26. 
médailles de l'empereur Alexandre I ${ }^{\text {er }}$, faisons l'hypothèse que c'est par son intermédiaire que les pierres gagnèrent la Russie.

Séduit par la beauté des minéraux ${ }^{83}$,E. A. Durand s'était doté d'un mobilier de pierre dure exécuté par des praticiens italiens et français : les catalogues des ventes de 1834 et 1836 témoignent du nombre et de la diversité de ces pièces massives. Il confiait en outre de remarquables pierres brutes aux célèbres tailleurs de gemmes ultramontains Filippo Rega, Giovanni Antonio Santarelli, Benedetto Pistrucci $^{84}$, Jean ${ }^{85}$ et Louis Pichler. On ne sait auquel des Pichler attribuer deux intailles : l'une vendue en $1836^{86}$, l'autre, «bague d'émeraude gravée représentant Vénus sortant du bain ${ }^{87}$ ", léguée par E. A. Durand à son ami E. J. Buquet, dont il est piquant de constater que ce dernier la lèguera à son tour, montée cette fois en épingle, à la baronne Attalin ${ }^{88}$.

\section{Un médaillier choisi}

L'intérêt d'E. A. Durand pour les médailles parait aussi précoce que durable : en 1817, son médaillier réunit 8371 médailles et en 1835, 1 432. Trois pistes d'approvisionnement sont envisageables. L'Espagne, d'abord, où l'ont mené ses affaires, et d'où il tient, sans doute, ses riches suites espagnoles et mauresques. L'Italie, ensuite, comme en témoignent notamment les trois as qu'il cède à la Bibliothèque et l'inventaire des caisses de ses dernières acquisitions. À Paris, enfin, achats et échanges devaient aller bon train entre les membres des cénacles numismatiques de Théodore-Edme Mionnet, employé puis conservateur de la Bibliothèque, et Charles-Louis Rollin, célèbre marchand numismate ${ }^{89}$.

Quelques indices révèlent un saut de qualité dans le médaillier Durand. La correspondance ayant accompagné l'achat par la Bibliothèque des pièces espagnoles du chevalier Durand en vante déjà le mérite ${ }^{90}$. De plus, la vingtaine de transactions effectuées avec la Bibliothèque révèle une inversion du rapport de forces vers 1812 : après avoir cédé numériquement plus qu’il ne recevait, E. A. Durand reçoit désormais davantage qu'il ne cède. En d'autres termes, de pourvoyeur en pièces de qualité relative, il devient fournisseur de pièces exceptionnelles ${ }^{91}$. Faisons l'hypothèse que, son intérêt pour la numismatique déclinant ${ }^{92}$, le chevalier Durand ait converti son médaillier auprès de la Bibliothèque, tantôt en numéraire, tantôt en spécimens plus courants, plus aisés à écouler dans sa boutique. Que H. K. E. Köhler ait acquis auprès d'E. A. Durand " une suite de médailles en or " pourrait le confirmer ${ }^{93}$.

\section{Le cabinet d'armes Percy-Durand}

Selon S. P. Chaudé, la formation du cabinet d'armes d'E. A. Durand conjurerait l'impression de vide éprouvée au lendemain de la vente de sa collection au Louvre en $1825^{94}$. Cette même année, le chevalier Durand acquiert le cabinet d'armes du baron Percy, chirurgien en chef des armées françaises, pour 60000 F., prévenant

83. S. P. Chaudé, op. cit. note 9, p. 9.

84. J. de Witte, op. cit. note $47, \mathrm{n}^{\circ} 2553$.

85. J. de Witte, op. cit. note $47, n^{\circ} 2527$.

86. J. de Witte, op. cit. note $47, \mathrm{n}^{\circ} 2643$.

87. Délivrance des legs, AN, ET/XXVI/1069.

88. Testament d'E. J. Buquet, AN, ET/XXVI/1131.

89. Charles Lenormant, « Nécrologie. M. Rollin », Revue de numismatique française, $\mathrm{n}^{\circ} 18$, 1853, pp. 162-163 et Thierry Sarmant, La République des médailles. Numismates et collections numismatiques à Paris du Grand Siècle au Siècle des Lumières, Paris, H. Champion, 2003, pp. 342-353.

90. T. Sarmant, Le cabinet des médailles de la Bibliothèque nationale 1661-1848, Paris, $\mathrm{H}$. Champion, 1994, p. 305 ; AN, F17/34/68.

91. Nous remercions Dominique Hollard de nous avoir fait prêter attention à cette dissymétrie. 92. E. A. Durand renonce par exemple à participer à I'achat du médaillier du comte de Wiczay que C. L. Rollin réalisera seul. Lettre d’E. A. Durand à C. L. Rollin, 27 septembre 1834, inventaire après décès cité note 14.

93. Lettre de H. K. E. Köhler à T. E. Mionnet, les 19-31 août 1822, BnF, NAF 1187, fo171.

94. S. P. Chaudé, op. cit. note 9, p. 12. 
ainsi la tenue d'une vente publique ${ }^{95}$. Son premier propriétaire, animé d'une passion pour les armes, a bénéficié de dons nombreux des belligérants côtoyés lors de sa carrière ${ }^{96}$. Le catalogue des trois cent quatre-vingts armes dont se composait cette collection témoigne d'un goût pour les " reliques » historiques, tels le casque du vizir ayant assiégé Vienne en 1783, offert par les chefs de la garde bourgeoise de Vienne ${ }^{97}$ ou la lance du hulan qui a tué La Tour d'Auvergne, offerte par le général Lecourbe ${ }^{98}$.

E. A. Durand poursuit l'entreprise du baron Percy, avec une prédilection pour la Renaissance, ainsi un pistolet de chasse et un bouclier d'Henri II ${ }^{99}$. Cependant, il se désintéresse bientôt de sa collection de militaria, pour laquelle il n'aurait pas eu de goût réel ${ }^{100}$. Reléguée dans un autre appartement, elle est vendue aux enchères du 18 au 23 janvier 1830. Faute de crédits suffisants, le musée d'artillerie n'y a pas fait d'acquisition ${ }^{101}$, mais reçoit, en deux occasions au moins, des pièces du cabinet Durand. À une date inconnue, d'abord, E. A. Durand fait don de vingt drapeaux et de la lance qui a tué La Tour d'Auvergne à cet établissement ${ }^{102}$. En 1839, ensuite, à l'occasion de la vente après décès du duc d'Istrie, la targe funéraire de Mathias Corvin les rejoint (fig. 5) ${ }^{103}$.
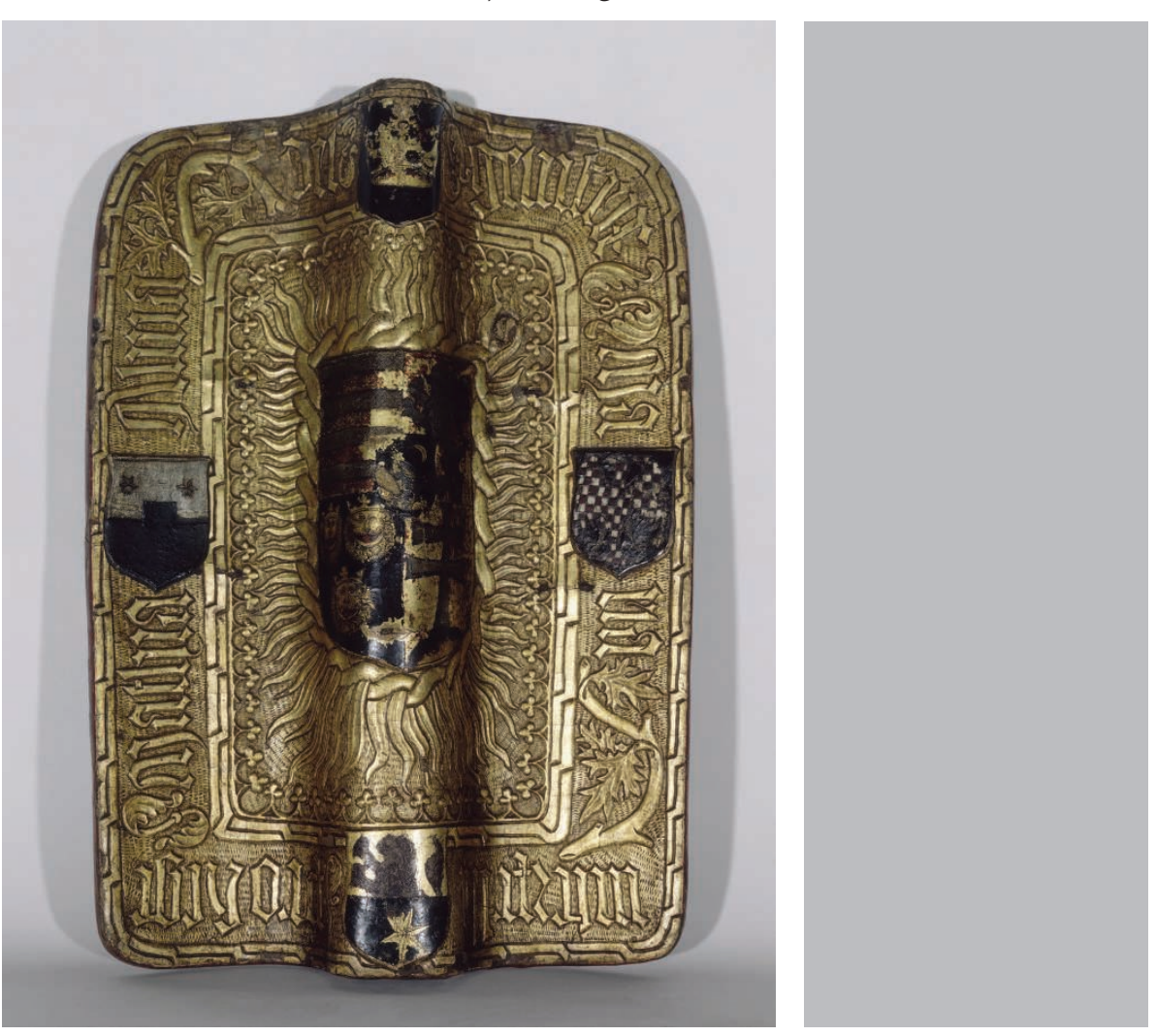

95. Léon Jean Joseph Dubois, Catalogue des antiquités, armures, armes, drapeaux et guidons (...) qui composaient la collection de feu M. le baron Percy, Paris, 1825, avis non paginé ; [Roussel ?], Catalogue des armures et armes diverses composant la collection formée originairement par feu M. le baron Percy, et complétée par M.D.***, Paris, 1829, p. 4.

96. L. J. J. Dubois, op. cit. note 95, p. I.

97. L. J. J. Dubois, op. cit. note $95, n^{\circ} 55$.

98. L. J. J. Dubois, op cit note $95, \mathrm{n}^{\circ} 89$.

99. [Roussel ?], op. cit. note $95, \mathrm{n}^{\circ} 317$ et 24

100. S. P. Chaudé, op. cit. note 9 , pp. $12-13$

101. Étienne Alexandre Bardin et Nicholas Charles Victor Oudinot, Dictionnaire de l'armée de terre, Paris, 1841, vol. II, article « cabinet d'armes ».

102. Inventaire après décès cité note 14 et liquidation de la succession citée note 11.

103. O. Penguilly L'Haridon, Catalogue des collections composant le musée d'artillerie, vol. III, 1862 , pp. 315-317 ; L. J. J. Dubois, op. cit. note $95, n^{\circ} 81$; [Roussel ?], op. cit. note $95, n^{\circ} 35$. 
La curiosité d'E. A. Durand excède largement le champ que délimitent les « deux » collections Durand. Aussi vaste que l'écrivait S. P. Chaudé, exigeante, à l'unisson de l'actualité de la recherche contemporaine, elle se nourrit du " grand amour de l'antiquité1 ${ }^{104}$ " que revendique le collectionneur. Mais la litanie des cabinets Durand trahit-elle un spéculateur avisé ou un érudit patriote ? Il nous semble que, collectionneur avant d'être homme d'affaires, E. A. Durand ait mis sa pratique du commerce au service de la collection d'œuvres d'art, avec un souci d'excellence qu'explique partiellement le désir de former des collections dignes des musées français ${ }^{105}$.

\section{L'auteur}

Élève conservateur, Institut national du patrimoine, Louise Detrez est diplômée de lettres classiques (licence, maîtrise) et ancienne élève de l'École du Louvre (premier et deuxième cycles). Dans son domaine de spécialité, la céramique grecque, elle s'intéresse notamment à l'histoire des collections et a consacré un article aux restaurateurs de vases antiques sollicités par le chevalier Durand (Technè, $\left.\mathrm{n}^{\circ} 38,2013\right)$. 